\title{
ERRATUM
}

Filino Harahap · Erfan Rudianto

IGD Mahendra Eka Pradnyana

\section{Measurements of steady-state heat dissipation from miniaturized horizontally-based straight rectangular fin arrays}

Published online: 11 November 2004

(C) Springer-Verlag 2004

Heat Mass Transfer (2004) DOI 10.1007/s00231-004-

0506-8

The definition for the Grashof number was incorrectly typeset: It should have been set as $\operatorname{Gr}\left(=g \beta \theta l^{3} / v^{2}\right)$. Likewise, equations (1) and (3) should read

$\mathrm{Nu}_{l, \mathrm{o}}=C\left[\operatorname{Gr}_{l, \mathrm{o}} \operatorname{Pr}_{\mathrm{o}}(n S / H)\right]^{a}(S / L)^{0.656}(H / L)^{0.412}$

$\mathrm{Nu}_{s, f}=C\left(\mathrm{Gr}_{s, f} \operatorname{Pr}_{f}\right)^{a}$

The online version of the original article can be found at http:// dx.doi.org/10.1007/s00231-004-0506-8

F. Harahap $(\square) \cdot$ E. Rudianto · IGD Mahendra Eka Pradnyana Thermal Engineering Laboratory, Mechanical Engineering

Department, Institute of Technology of Bandung (ITB),

Jl. Ganesha 10, Bandung 40132, Indonesia

E-mail: filhap@attglobal.net 